\title{
Simple Thrust Formula for an MPD Thruster with Applied- Magnetic Field from Magnetic Stress Tensor
}

\author{
M. Coletti* \\ University of Southampton, Southampton, United Kingdom
}

\begin{abstract}
It has been previously shown that the application of an axial magnetic field to an MPD thruster increases the thrust and focuses the plasma jet. The goal of this paper is to obtain a simple and ready-to-use theory to predict the thrust generated by an applied-field MPD. To derive a simple thrust formula some assumptions have been made: the applied field is assumed to be purely axial inside the thruster and the total current, plasma temperature, mass flow rate and axial velocity at the exit section of anode to be not sensibly influenced by the application of the magnetic field. Using the magnetic stress tensor the forces acting on the plasma inside the thruster can be derived. Assuming that the detachment from the magnetic nozzle outside the MPD happens when the charged particle motions violate the adiabatic condition the thrust expression can be finally derived. Comparison with experimental data confirms these hypotheses and gives good agreement with theory.
\end{abstract}

\section{Nomenclature}

$=$ magnetic field

$=$ magnetic stress tensor

$=$ energy

$=$ force

$=$ force per unit volume

$=$ thrust

$=$ current

$=$ moment of inertia of the plasma column

$=$ current in the magnetic coil

$=$ current density

$=$ anode length

$=$ mass of the plasma column inside the thruster

$=$ ionic mass

$=$ mass flow rate

$=$ torque

$=$ ion density, normal vector

$=$ pressure

$=$ Teflon gas constant

$=$ magnetic coil radius

$=$ radius

$=$ surface

$=$ temperature

$=$ volume

$=$ velocity

$=$ cylindrical coordinate

$=$ angular velocity

= vacuum magnetic permeability

\footnotetext{
${ }^{*} \mathrm{PhD}$ student, Astronautic Research Group, School of Engineering Sciences, University of Southampton, SO17 1BJ Southampton U.K. coletti@soton.ac.uk. This paper is taken from the Author MSc final dissertation ${ }^{1}$ held in the Scuola di Ingegneria Aerospaziale of the University of Rome "La Sapienza" in October 2005, Rome, Italy
} 


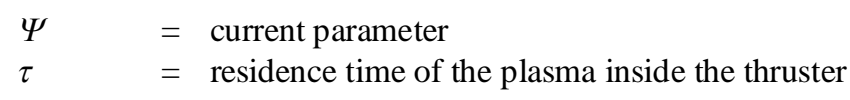

Subscript

$a=$ relative to the anode

$B \quad=$ relative to the magnetic field

$B=0 \quad=$ without applied field

$c=$ relative to the cathode

$e \quad=$ at the exit of the thrusters

$\exp \quad=$ experimental

$f \quad=$ at the end of the magnetic nozzle

$i=$ at the beginning of the magnetic nozzle

$J \quad=\quad$ relative to the plasma jet with applied field

$L=$ Larmor

$t \quad=$ relative to the cylindrical surface of the anode

th $=$ thermodynamic

theo $=$ theoretical

$z, \theta, r=$ direction

$0=$ on the exit surface of the anode

$\perp \quad=$ perpendicular to magnetic field lines

Superscripts

$\begin{array}{lll}\rightarrow & = & \text { vector } \\ \wedge & = & \text { unit vector }\end{array}$

\section{Introduction}

I OW thrust propulsive systems have a very important role in the accomplishment of a space mission. For these systems electric propulsion is more suitable than chemical one due to its higher specific impulse.

One of the most important and most studied electric thrusters is the Magneto Plasma Dynamic thruster. Significant effort has been made in studying the effect that an externally applied magnetic field has on an MPD thruster's performance and how to predict these effects.

Dealing with the thrust developed by this thruster when a magnetic field is externally applied, three theories were developed respectively by Fradkin ${ }^{2}$, Sasoh ${ }^{3}$ and Turchi ${ }^{4}$.

Sasoh's and Turchi's theories are exhaustive and comprehensive but while Sasoh's does not lend itself to simple calculations Turchi's one depends on the knowledge of the plasma viscosity that is not easy to determine. Fradkin's theory, instead, is simple and easy to use but gives results that do not match well the experimental observation.

The purpose of this work is to develop an easy-to-use theory to predict the thrust generated by an applied-field MPD thruster and compare it with the experimental data.

\section{Hypothesis}

To derive a simple thrust formula some hypotheses must be done. The applied field is assumed to be purely axial inside the thruster, the field's lines to diverge only outside it and the applied field to be much stronger than the self induced one.

The total current $I$, plasma temperature $T$, mass flow $\dot{m}$ and axial velocity $v_{z 0}$ at the exit section of anode are assumed not to be sensibly influenced by the application of the magnetic field. The gasdynamic thrust will be neglected in comparison with the electromagnetic one, so that thrust with no external-applied field is ${ }^{5}$

$$
\mathrm{F}_{B=0}=\frac{\mu I^{2}}{4 \pi}\left(\ln \left(\frac{r_{a}}{r_{c}}\right)+\frac{3}{4}\right)
$$

and that the exhaust velocity with no applied field is 


$$
v_{z 0}=\frac{\mathrm{F}_{B=0}}{\dot{m}}=\frac{\mu I^{2}}{4 \pi \dot{m}}\left(\ln \left(\frac{r_{a}}{r_{c}}\right)+\frac{3}{4}\right)
$$

\section{Effect of the Applied Magnetic Field Inside the MPD Thruster}

The effect of an applied magnetic field is to produce a swirling motion in the plasma jet and to focus it on to the thruster axis. The swirling energy is then converted into axial energy, increasing the thrust, by the magnetic nozzle created by the diverging field's line out of the thruster.

To study the problem the magnetic stress tensor will be used ${ }^{1,6}$. Taking an MPD with a cylindrical anode and cathode with radius respectively $r_{a}$ and $r_{c}$, is convenient to use a cylindrical reference system $r, \theta, z$

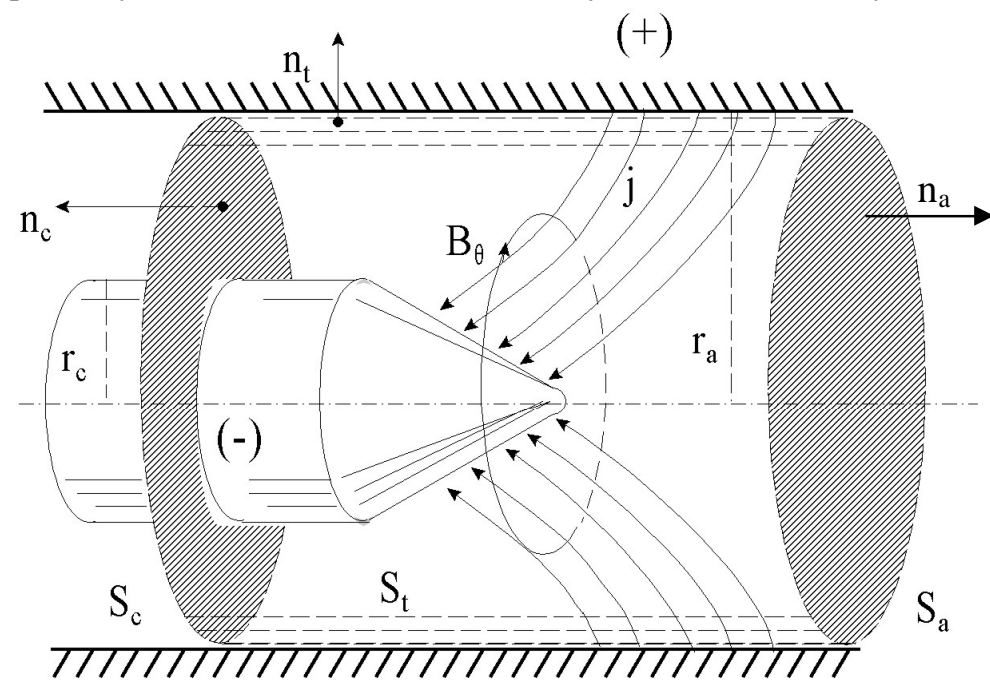

$(+)$

Figure 1. Thruster geometry and integration surface

The forces acting on plasma are Lorentz's forces and can be expressed as

$$
\vec{F}=\int_{V} \vec{f} d V=\int_{V} \vec{j} \times \vec{B} d V
$$

using Maxwell's equation $\nabla \times \vec{B}=\mu \vec{j}$ Eq (3) becomes

$$
\vec{f}=\vec{j} \times \vec{B}=\frac{1}{\mu}(\nabla \times \vec{B}) \times \vec{B}
$$

introducing the magnetic stress tensor $\mathbf{B}$

$$
\frac{1}{\mu}(\nabla \times \vec{B}) \times \vec{B}=\nabla \cdot \mathbf{B}-\frac{1}{\mu} \vec{B} \nabla \cdot \vec{B}
$$

using Maxwell's equation $\nabla \cdot \vec{B}=0$ and the divergence theorem we finally obtain

$$
\vec{F}=\int_{V} \vec{j} \times \vec{B} d V=\int_{V} \nabla \cdot \mathbf{B} d V=\int_{S} \mathbf{B} \cdot \hat{n} d V
$$

Using Eq (6) the forces acting on plasma are expressed like the flux of the tensor $\mathbf{B}$ through the boundary surface of the volume $V$ representing the thruster. Hence, assuming a cylindrical geometry for the MPD, the control surface looks like the one drawn in Fig 1.

It can be demonstrated that the magnetic stress tensor in cylindrical coordinates has the following expression ${ }^{1}$ 


$$
\mathbf{B}=\frac{1}{\mu}\left[\begin{array}{ccc}
B_{r}^{2}-\frac{B^{2}}{2} & \frac{B_{r} B_{\theta}}{r} & B_{r} B_{z} \\
\frac{B_{\theta} B_{r}}{r} & B_{\theta}^{2}-\frac{B^{2}}{2} & \frac{B_{\theta} B_{z}}{r} \\
B_{z} B_{r} & \frac{B_{z} B_{\theta}}{r} & B_{z}^{2}-\frac{B^{2}}{2}
\end{array}\right]
$$

In the case of study the only non zero component of the magnetic field is $B_{\theta}$ (the self induced magnetic field) and $B_{z}$ (the externally applied magnetic field); so the stress tensor becomes

Hence the force per unit of surface is

$$
\mathbf{B}=\frac{1}{\mu}\left[\begin{array}{ccc}
-\frac{B_{\theta}^{2}}{2}-\frac{B_{z}^{2}}{2} & 0 & 0 \\
0 & -\frac{B_{\theta}^{2}+B_{z}^{2}}{2 r^{2}} & \frac{B_{\theta} B_{z}}{r} \\
0 & \frac{B_{z} B_{\theta}}{r} & \frac{B_{z}^{2}}{2}-\frac{B_{\theta}^{2}}{2}
\end{array}\right]
$$

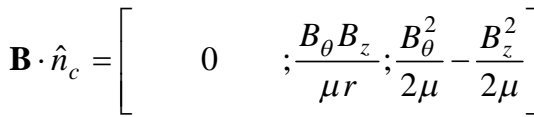

$$
\begin{aligned}
& \mathbf{B} \cdot \hat{n}_{t}=\left[-\frac{B_{\theta}^{2}}{2 \mu}-\frac{B_{z}^{2}}{2 \mu} ; 0 \quad ; \quad 0\right] \\
& \mathbf{B} \cdot \hat{n}_{a}=\left[\begin{array}{lll}
0 & ; 0 & ; \frac{B_{z}^{2}}{2 \mu}-\frac{B_{\theta}^{2}}{2 \mu}
\end{array}\right]
\end{aligned}
$$

On the anode surface $S_{a}$ the axial current is zero so also $B_{\theta}$ is zero, thus the forces acting on plasma are

$$
\begin{aligned}
& F_{r}=-\int_{S_{t}} \frac{B_{\theta}^{2}}{2 \mu}+\frac{B_{z}^{2}}{2 \mu} d S \\
& F_{\theta}=\int_{S_{c}} \frac{B_{\theta} B_{r}}{\mu r} d S \\
& F_{z}=\int_{S_{a}} \frac{B_{\theta}^{2}}{2 \mu} d S
\end{aligned}
$$

the term $\frac{B_{\theta}^{2}}{2 \mu}$ in the radial and axial component of the force represent the pumping and blowing contributions of Maecker's formula ${ }^{5}$.

Integrating $F_{\theta}$ we obtain

$$
F_{\theta}=B_{z} I\left(\ln \frac{r_{a}}{r_{c}}+\frac{1}{2}\right)
$$

It can be noted that this is not a pure force but a force per unit of length so it gives rise to an angular acceleration.

Supposing that the plasma rotates like a rigid body, to determine its angular velocity $F_{\theta}$ must be integrated along the ion's trajectory; unfortunately this trajectory is unknown so $\omega$ can not be determined this way.

The torque generated by $F_{\theta}$ can be expressed as

$$
M=F_{\theta} \tilde{r}^{2}=I_{i n} \frac{\omega}{\tau}=m \tilde{r}^{2} \frac{\omega}{\tau}
$$

where here $m$ represents the mass of the plasma column inside the thruster and $\tilde{r}$ is a mean value of the radius such that 


$$
I_{i n}=\int_{0}^{r_{a}} \rho r^{2} 2 \pi r d r=m \tilde{r}^{2}
$$

Noting that the mass flow rate is equal to

$$
\dot{m}=\frac{m}{\tau}
$$

the angular velocity of plasma can be expressed as

Now integrating $F_{r}$ we obtain

$$
\omega=\frac{F_{\theta}}{\dot{m}}=\frac{B_{z} I}{\dot{m}}\left(\ln \frac{r_{a}}{r_{c}}+\frac{1}{2}\right)
$$

$$
F_{r}=-\frac{B_{\theta}^{2}+B_{z}^{2}}{2 \mu} 2 \pi r_{a} L_{a}
$$

this force is direct radially inward and distributed over the surface $S_{t}$ so can be represented as a pressure. Assuming that the applied field is much stronger than the self-induced one $B_{\theta}{ }^{2}$ can be neglected and so the pressure becomes

$$
p_{B}=\frac{B_{z}^{2}}{2 \mu}
$$

This pressure must be balanced by the thermodynamic pressure of plasma. The thermodynamic pressure inside the plasma is

$$
p_{t h}=2 n k_{B} T
$$

Assuming that with no applied magnetic field the plasma jet have the same dimension of the anode (that means plasma fills all the available space on exit surface) $n_{B=0}$ can be inferred from the mass conservation equation.

$$
\begin{gathered}
\dot{m}=\rho v_{z 0} S_{a}=m_{i} n_{B=0} v_{z 0} \pi r_{a}^{2} \\
\Downarrow \\
n_{B=0}=\frac{\dot{m}}{m_{i} v_{z 0} \pi r_{a}^{2}}
\end{gathered}
$$

Now writing and combining the mass conservation equation for the applied and not-applied magnetic field case one relation for the charge density $n$ can be obtained

$$
\begin{gathered}
\dot{m}=m_{i} n_{B=0} v_{z 0} \pi r_{a}^{2} \quad \dot{m}=m_{i} n v_{z 0} \pi r_{J}^{2} \\
n=n_{B=0} \frac{r_{a}^{2}}{r_{J}^{2}}
\end{gathered}
$$

Imposing now that the thermodynamic pressure $p_{t h}$ balance $p_{B}$ the dimension of plasma jet with applied magnetic field $r_{J}$ can be obtained

$$
\begin{aligned}
& p=p_{B} \\
& 2 n_{B=0} \frac{r_{a}^{2}}{r_{J}^{2}} k_{B} T=-\frac{B_{\theta}^{2}+B_{z}^{2}}{2 \mu} \approx-\frac{B_{z}^{2}}{2 \mu} \\
& r_{J}=\sqrt{\frac{4 \mu n_{B=0} k_{B} T}{B_{z}^{2}}} r_{a}
\end{aligned}
$$

now substituting $n_{B=0}$ in Eq (19) and using expression (2) for the exit velocity $v_{z 0}$ the final expression for $r_{J}$ becomes

$$
r_{J}=\sqrt{\frac{4 \mu \dot{m}^{2} k_{B} T}{\pi m_{i} \mathrm{~F}{ }_{B=0} B_{z}^{2}}}
$$

\section{Effect of the Applied Magnetic Field Outside the MPD Thruster: the Magnetic Nozzle}

Outside the MPD thruster the magnetic field's lines start to diverge giving rise to a magnetic nozzle. 


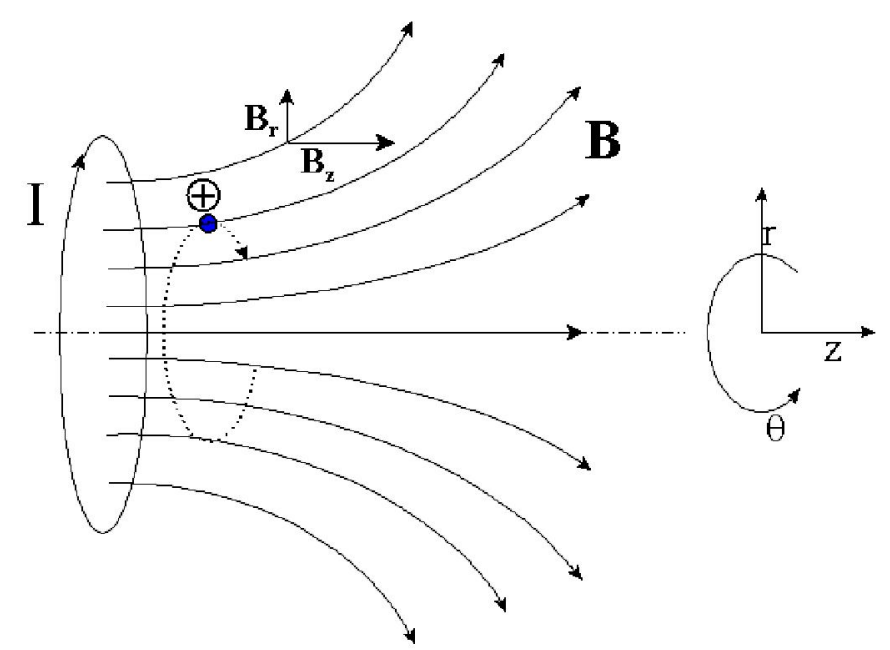

Figure 2. Magnetic Nozzle

The effect of the magnetic nozzle is mainly to transfer kinetic energy of a particle from its motion perpendicular to magnetic field's lines towards the one parallel to them. This transfer could be expressed with the following law:

$$
\frac{v_{\perp i}^{2}}{B_{i}}=\frac{v_{\perp f}^{2}}{B_{f}}
$$

this transfer lasts until the scale of the swirling motion perpendicular to the field's lines is much smaller than the scale of the variation of the field, that is

$$
\frac{\|\nabla \vec{B}\|}{B} r_{L}<<1
$$

For our purpose is extremely important to know where the particle "detaches" from the magnetic nozzle, and so where the energy transfer ends, to calculate of much swirling energy is converted into axial energy and so in thrust.

Arbitrarily supposing that the transfer ends when

$$
\frac{\|\nabla \vec{B}\|}{B} r_{L}=1
$$

and that this happens so far from the magnetic field source to consider it like a magnetic dipole the magnetic field component and the magnetic field strength can be expressed like

$$
\begin{aligned}
& B_{r}=\frac{\mu I_{m} R_{m}^{2}}{4} \frac{3 z r}{\left(z^{2}+r^{2}\right)^{/ 2}} \\
& B_{z}=\frac{\mu I_{m} R_{m}^{2}}{4} \frac{2 z^{2}-r^{2}}{\left(z^{2}+r^{2}\right)^{/ 2}} \\
& B=\frac{\mu I_{m} R_{m}^{2}}{4} \frac{\sqrt{4 z^{2}+r^{2}}}{\left(z^{2}+r^{2}\right)^{2}}
\end{aligned}
$$

Expressing ||$\nabla \vec{B}||$ like $|\nabla \vec{B} \cdot \hat{B}|$, substituting into (25) and supposing that when a particle detaches it is sufficiently near to the axis to neglect $r^{2}$ terms in comparison with $z^{2}$ the detachment point coordinate is obtained

$$
\begin{gathered}
\bar{z}=\left(\sqrt{\mu I_{m} R_{m}^{2}} \frac{1}{3 \sqrt{2}} \sqrt{B_{i}} \frac{q}{v_{\perp i} m_{i}}\right)^{2}=\frac{\alpha}{v_{\perp i}^{2}} \\
\alpha=\left(\sqrt{\mu I_{m} R_{m}^{2}} \frac{1}{3 \sqrt{2}} \sqrt{B_{i}} \frac{q}{m_{i}}\right)^{2}
\end{gathered}
$$


Now using expression (23) and substituting (27) into the third of (26) remembering that $\bar{z}^{2}>\bar{r}^{2}$ the residual velocity perpendicular to field's lines is

$$
v_{\perp f}=v_{\perp i} \sqrt{\frac{\bar{B}}{B_{i}}}=v_{\perp i}^{4} \sqrt{\frac{R_{m}^{3}}{\alpha^{3}}}
$$

Noting now that the kinetic energy is conserved the axial velocity at the exit of the magnetic nozzle is

$$
v_{z f}=\sqrt{v_{0}^{2}-v_{\perp i}^{8} \frac{R_{m}^{3}}{\alpha^{3}}}
$$

\section{Thrust formula}

Thrust could be generally expressed like

$$
\mathrm{F}=\dot{m} v_{z f}
$$

in our case $v_{z f}$ is a function of $r$ so a mean value of the velocity must be used. It can be defined as

$$
\bar{v}_{z f}=\frac{\int_{A} v_{z f} d A}{\int_{A} d A}=\frac{\int_{0}^{r_{J}} \sqrt{v_{z 0}^{2}+\omega^{2} r^{2}-\omega^{8} r^{8} \frac{R_{m}^{3}}{\alpha^{3}}} r d r}{\int_{0}^{r_{J}} r d r}
$$

unfortunately this integral can not be solved analytical. Looking at the trend of $v_{z f}$ it can be noted that it is very similar to a parabola so it can be interpolated with a second order polynomial

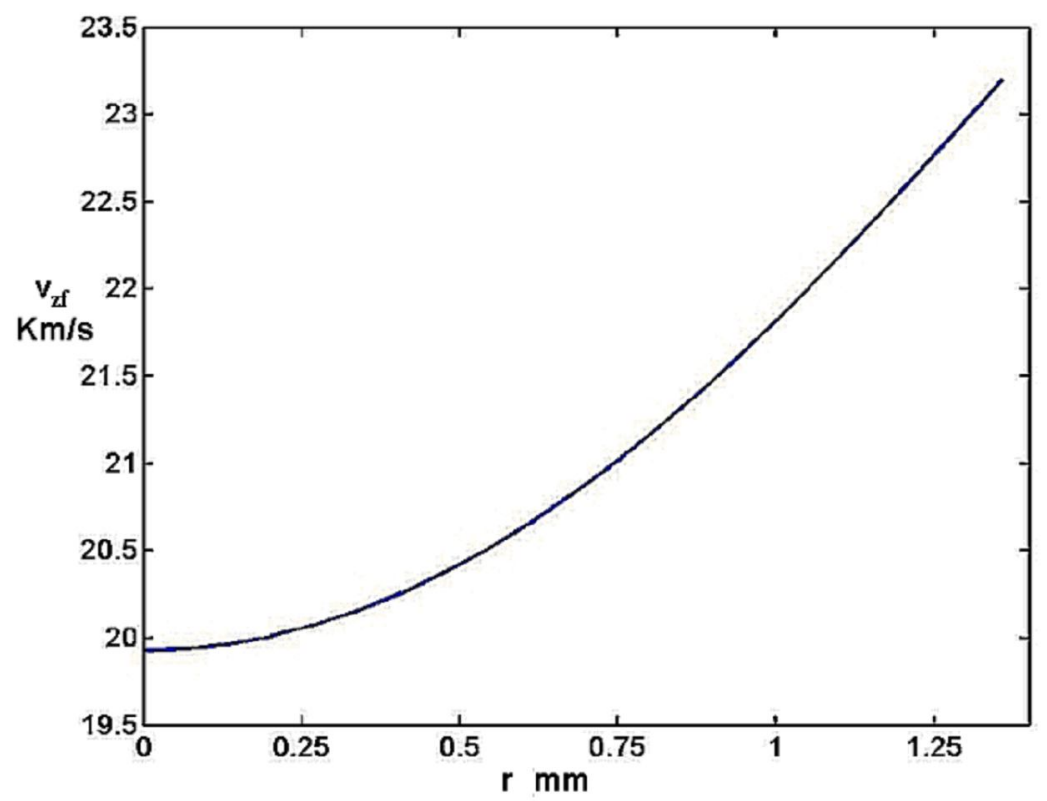

Figure 3. Trend of $v_{z f}$ with $r \omega=8.9 \cdot 10^{6} \alpha=4.43 \cdot 10^{8}$

using $r=0$ and $r=r_{J}$ as interpolation points 


$$
\begin{aligned}
& v_{z f}=a r^{2}+b \\
& a=\frac{-v_{z 0}+\sqrt{v_{z 0}^{2}+\omega^{2} r_{J}^{2}-\omega^{8} r_{J}^{8} \frac{R_{m}^{3}}{\alpha^{3}}}}{r_{J}^{2}} \\
& b=v_{z 0}
\end{aligned}
$$

substituting into Eq (31) the mean exit velocity is obtained

$$
\bar{v}_{z f}=\frac{\int_{A} \bar{v}_{z f} d A}{\int_{A} d A}=\frac{\int_{0}^{r_{J}}\left(a r^{2}+b\right) d r}{\int_{0}^{r_{J}} r d r}=a \frac{r_{J}^{2}}{2}+b
$$

hence using Eq (30) the thrust can be finally expressed as

$$
\mathrm{F}=\dot{m}\left(a \frac{r_{J}^{2}}{2}+b\right)=\dot{m}\left(a \frac{r_{J}^{2}}{2}+v_{z 0}\right)=\mathrm{F}_{B=0}+\dot{m} a \frac{r_{J}^{2}}{2}
$$

\section{Comparison with experimental results and conclusions}

A comparison between the theory developed above and experimental results is made using old data from the Propulsion Laboratory of the University of Rome "La Sapienza" relative to the MIRA thruster. Below is a table reporting for each energy level the current parameter $\Psi$, the temperature $T$, the mass flow rate, the thrust with and without applied field and its theoretical value.

\begin{tabular}{|c|c|c|c|c|}
\hline$E[\mathrm{~J}]$ & 14359 & 16397 & 18672 & 21541 \\
\hline$\Psi\left[\mathrm{A}^{2} \mathrm{~s}\right]$ & 42684 & 51473 & 60602 & 73339 \\
\hline$\dot{m}[\mathrm{~g} / \mathrm{s}]$ & 0.524 & 0.591 & 0.732 & 0.892 \\
\hline$T[\mathrm{eV}]$ & 0.93 & 1.7 & 2.55 & 3.63 \\
\hline$F_{\mathrm{B}=0}[\mathrm{~N}]$ & 10.3 & 12.31 & 14.03 & 16.13 \\
\hline $\mathrm{F}_{\text {exp }}[\mathrm{N}]$ & 11.78 & 13.66 & 16.2 & 19.05 \\
\hline $\mathrm{F}_{\text {theo }}[\mathrm{N}]$ & 10.83 & 13.30 & 15.87 & $0.6 \%$ \\
\hline error & $8.5 \%$ & $2.6 \%$ & $2 \%$ & \\
\hline
\end{tabular}

As can be noted there is a good agreement between theory and experiments with errors of some percent. The fact that the higher the energy the smaller is the error could be expected because the higher the energy the more accurate is the hypothesis that gasdynamics effects are neglegible.

At present new measurements are ongoing to confirm all the other hypotheses. The data obtained up to now seem to verify the hypothesis and will be published at the next IEPC in Florence.

\section{Acknowledgments}

The author want to thank Prof Paccani for the support given during all the work done in the University of Rome "La Sapienza" and to thank all that people that help him in these last three years.

\section{References}

${ }^{1}$ Coletti M., "La Spinta Nei Propulsori MPD con Campo Magnetico Applicato”, Master Thesys, Scuola di Ingegneria Aerospaziale, University of Rome "La Sapienza”, Rome, Italy, October 2005 
AIAA - 2007 - 5284

${ }^{2}$ Fradkin D.B.,"Analysis of Acceleration Mechanisms and Performance of an Applied Field MPD Arcjet”, Ph.D. Dissertation, Princeton University MAE Dept, 1973

${ }^{3}$ Sasoh A., Arakawa Y., "Electromagnetic Effects in an Applied-Field Magnetoplasmadynamic Thruster", Journal of Propulsion, Vol 8, No 1, 1992

${ }^{4}$ Mikellides P.G., Turchi P.J., "A theoretical model for the thrust and voltage of applied-field MPD thrusters", AIAA-19983474, AIAA/ASME/SAE/ASEE Joint Propulsion Conference and Exhibit, 34th, Cleveland, OH, July 13-15, 1998

${ }^{5}$ H. Maecker. Z. Phys., 141(1):198-216, 1955.

${ }^{6}$ Jahn R.G., "Physics of Electric Propulsion", McGraw-Hill, New York, 1968.

${ }^{7}$ Kuriki K., Okada O.: "Experimental Study of Plasma Flow in a Magnetic Nozzle”, The Physics of Fluids, Vol 13, No 9, September 1970

${ }^{8}$ Okada O., Kuriki K.: "The Interaction between a Plasma Flow and a Magnetic Nozzle with Strong Hall Effect", ISAS Report No 457, Vol 35, No 15, Dicember 1970

${ }^{9}$ Seshadri S.R.: "Fundamentals of Plasma Physics", American Elsevier Publishing Company Inc., New York, 1973

${ }^{10}$ Chen F.F. : "Introduction to Plasma Physics" , Plenum Press, New York, 1974

${ }^{11}$ Kimura I., Arakawa Y.: "Effects of Applied Magnetic Fields on Physical Processes in an MPD Arcjet", AIAA-76-1001, AIAA International Electric Propulsion Conference, Key Biscayne (Florida), November 1976

${ }^{12}$ Tahara H., Kagaya Y., Yoshikawa T.: "Quasi-Steady MPD Arcjets with Applied Magnetic Fields”, AIAA-85-2001, 18 $8^{\text {th }}$ International Electric Propulsion Conference, Alexandria (Virginia), September 1985

${ }^{13}$ Arakawa Y., Sasoh A.: "Steady-State Permanent Magnet MPD Thruster", AIAA-87-1021, 19th International Electric Propulsion Conference, Colorado Springs (Colorado), May 1987

${ }^{14}$ Arakawa Y., Sasoh A.: "Steady-State Permanent Magnet Magnetoplasmadynamic Thruster", Journal of Propulsion, Vol 5, No 3,May- June, 1989

${ }^{15}$ Myers R. M., Mantenieks M., Sovey J. : “Geometric Effetcs in Applied-Field MPD Thruster”, AIAA-90-2669 21st International Electric Propulsion Conference, Orlando (Florida), July 1990 